\title{
A one-person doxastic characterization of Nash strategies
}

\author{
Andrés Perea
}

Received: 22 February 2007 / Accepted: 26 June 2007 / Published online: 21 July 2007

(C) Springer Science+Business Media B.V. 2007

\begin{abstract}
Within a formal epistemic model for simultaneous-move games, we present the following conditions: (1) belief in the opponents' rationality (BOR), stating that a player believes that every opponent chooses an optimal strategy, (2) selfreferential beliefs (SRB), stating that a player believes that his opponents hold correct beliefs about his own beliefs, (3) projective beliefs (PB), stating that $i$ believes that $j$ 's belief about $k$ 's choice is the same as $i$ 's belief about $k$ 's choice, and (4) conditionally independent beliefs (CIB), stating that a player believes that opponents' types choose their strategies independently. We show that, if a player satisfies BOR, SRB and CIB, and believes that every opponent satisfies BOR, SRB, PB and CIB, then he will choose a Nash strategy (that is, a strategy that is optimal in some Nash equilibrium). We thus provide a sufficient collection of one-person conditions for Nash strategy choice. We also show that none of these seven conditions can be dropped.
\end{abstract}

Keywords Nash equilibrium · Epistemic game theory

\section{Introduction}

Since its introduction by Nash (1951), the concept of Nash equilibrium has played an essential role in game theory and its various applications. It is therefore natural to look for reasonable conditions under which players may be expected to choose according to Nash equilibrium. Such conditions have been provided in many different settings. In learning theory one assumes that players play the game repeatedly, and one can find

\footnotetext{
A. Perea $(\varangle)$

Department of Quantitative Economics, Maastricht University, P.O. Box 616, Maastricht, 6200 MD, The Netherlands

e-mail: a.perea@ke.unimaas.nl

http://www.personeel.unimaas.nl/a.perea/
} 
reasonable classes of learning rules that eventually lead players to choose according to some Nash equilibrium. Similarly, evolutionary game theory studies classes of replicator dynamics in the repeated game that converge to (special types of) Nash equilibria in the long run. If the game is to be played only once, one could think of a situation in which a mediator publicly announces the mixed strategy profile to the players, and recommends each player to play his strategy in this profile. Then, players may only be expected to follow their recommendation if the mixed strategy profile is a Nash equilibrium. One can also formulate sufficient conditions for Nash equilibrium in a static setting without mediators. See, for instance, Brandenburger and Dekel (1987), Aumann and Brandenburger (1995) and Asheim (2006). A key condition in each of these models is that a player knows, or has a correct belief about, his opponents' beliefs about the other players' strategy choices.

A common feature of each of the models above is that it requires, either explicitly or implicitly, some sort of communication between players. In the learning models and evolutionary models players communicate by the actions they choose in the repeated game. It is this type of communication that allows them to converge to a Nash equilibrium in the long run. In the static models where players are assumed to have correct beliefs about the other players' beliefs, there seems to be a need for ex-ante communication between players in which they report their beliefs to others. Otherwise, there is no reason to expect that players should be right about the opponents' beliefs, even if common belief in rationality is imposed.

In this paper, we completely focus on static settings in which there is no communication between players. In such a setting, a player can base his strategy choice solely upon his own beliefs about the opponents' choices and his own beliefs about the opponents' beliefs, since before making his decision he receives no information about what opponents do or believe. It thus makes sense to analyze the game from a single player's perspective, and this is exactly what we will do. More precisely, we focus on one player, say player $i$, in the game, and impose conditions solely on the beliefs that player $i$ has about the other players' strategy choices and the other players' beliefs. We do not impose any conditions on the beliefs and choices of players other than $i$. We thus allow for the event that player $i$ believes that some opponent believes or chooses in a certain way, whereas in fact this opponent believes or chooses differently.

A question that arises within this one-person approach is: "How should we interpret Nash equilibrium?" Usually, Nash equilibrium is either interpreted as an equilibrium in choices or as an equilibrium in beliefs. The first interpretation states that a player's choice should be optimal given the other players' choices, whereas the second interpretation states that player $i$ 's belief about player $j$ 's choice should only assign positive probability to choices that are optimal for player $j$, given $j$ 's belief about the other players' choices. Both interpretations, however, require that we simultaneously impose conditions on the choices or beliefs of all players, not just player $i$. So, how can we make sense of Nash equilibrium in a one-person approach?

A possible way is to interpret all the beliefs in a Nash equilibrium as belonging to a single player. Consider, for instance, a game with two players, say players $i$ and $j$, and a Nash equilibrium $\left(\mu_{i}, \mu_{j}\right)$, where $\mu_{i}$ is a probability distribution over $i$ 's choices, and $\mu_{j}$ is a probability distribution over $j$ 's choices. A possible one-person interpretation is that $\mu_{j}$ represents $i$ 's belief about $j$ 's choice, and that $\mu_{i}$ represents $i$ 's belief 
about $j$ 's belief about $i$ 's choice. That is, the Nash equilibrium $\left(\mu_{i}, \mu_{j}\right)$ is completely "in the mind of player $i$," as $\mu_{i}$ and $\mu_{j}$ represent beliefs that both belong to him. The Nash equilibrium, when interpreted in this way, says nothing about player $j$ 's actual belief about $i$ 's choice. In fact, it may be the case that in this Nash equilibrium, player $i$ is completely wrong about what $j$ really believes or does.

Once we interpret Nash equilibrium as describing player $i$ 's state of mind, we may explore its behavioral consequences. So, again in a two player setting, if $i$ 's belief about $j$ 's choice and $i$ 's belief about $j$ 's belief about $i$ 's choice together constitute a Nash equilibrium, what can $i$ rationally choose? The answer is almost tautological: any strategy choice for $i$ that is optimal in some Nash equilibrium $\left(\mu_{i}, \mu_{j}\right)$. We refer to such strategies as Nash strategies. The logical relation between Nash strategies on the one hand, and strategies receiving positive probability in some Nash equilibrium on the other hand is more subtle than it may appear at first. Every strategy that receives positive probability in some Nash equilibrium is a Nash strategy, but not every Nash strategy receives positive probability in some Nash equilibrium. Consider, for instance, the game in Fig. 1. Player 1 is the row player, and player 2 the column player. In this game, $\left(b, \frac{1}{2} c+\frac{1}{2} d\right)$ is a Nash equilibrium. Since strategy $a$ is optimal against $\frac{1}{2} c+\frac{1}{2} d$, it follows that $a$ is a Nash strategy. However, there is no Nash equilibrium $\left(\mu_{1}, \mu_{2}\right)$ in which $\mu_{1}$ assigns positive probability to $a$. At the same time, we should not exclude the choice $a$ if we require that player 1's belief about player 2's choice and player 1's belief about player 2's belief about his own choice constitute a Nash equilibrium. It is possible, namely, that player 1's belief about 2's choice is given by $\frac{1}{2} c+\frac{1}{2} d$, that he believes that 2 believes that he will choose $b$, whereas in fact he will choose $a$. Then, player 1's beliefs above constitute a Nash equilibrium, but he chooses a Nash strategy that cannot have positive probability in any Nash equilibrium.

The objective of this paper is to identify reasonable conditions on the beliefs of a single player which would guarantee that this player eventually chooses a Nash strategy. We thus offer one-person doxastic conditions for Nash strategies. As an illustration of our objective, consider the game in Fig. 2, which is taken from Myerson (1991,

\begin{tabular}{|l|l|l|}
\hline & $c$ & $d$ \\
\hline$a$ & 2,0 & 0,2 \\
\hline$b$ & 1,1 & 1,1 \\
\hline
\end{tabular}

Fig. 1 Not every Nash strategy is assigned positive probability in a Nash equilibrium

\begin{tabular}{|l|l|l|l|}
\hline & $d$ & $e$ & $f$ \\
\hline$a$ & 3,0 & 0,2 & 0,3 \\
\hline$b$ & 2,0 & 1,1 & 2,0 \\
\hline$c$ & 0,3 & 0,2 & 3,0 \\
\hline
\end{tabular}

Fig. 2 Rationalizability versus Nash strategies 
p. 94). Suppose that the game is played only once, and that player 1 and player 2 cannot communicate to each other. We will analyze this game completely from player 1 's perspective. It can be shown that common belief in rationality does not exclude any of player 1's strategies, but that strategy $b$ is the only Nash strategy for player 1 . Consider, namely, a scenario in which

1. player 1 believes that player 2 chooses $d$,

2. player 1 believes that player 2 believes that player 1 chooses $c$,

3. player 1 believes that player 2 believes that player 1 believes that player 2 chooses $f$,

4. player 1 believes that player 2 believes that player 1 believes that player 2 believes that player 1 chooses $a$,

5. player 1 believes that player 2 believes that player 1 believes that player 2 believes that player 1 believes that player 2 chooses $d$,

and so on. Then, player 1 respects common belief in rationality, since he believes that player 2 chooses rationally, believes that player 2 believes that player 1 chooses rationally, and so on. Since in this scenario it is rational for player 1 to choose $a$, we must allow for the strategy $a$ if we only impose common belief in rationality. Since strategies $a$ and $c$ play similar roles in this game, one can construct a similar scenario that leads to strategy choice $c$. Finally, strategy $b$ can be justified by a much simpler scenario in which

1. player 1 believes that player 2 chooses $e$,

2. player 1 believes that player 2 believes that player 1 chooses $b$,

3. player 1 believes that player 2 believes that player 1 believes that player 2 chooses $e$,

and so on. Summarizing, every strategy for player 1 can be justified by a belief hierarchy for player 1 that respects common belief in rationality. More generally, Tan and Werlang (1988) have shown that the strategies which can be chosen rationally in two-player games when imposing only common belief in rationality are exactly the rationalizable strategies in the sense of Bernheim (1984) and Pearce (1984). ${ }^{1}$

On the other hand, it can be shown that $b$ is the only Nash strategy for player 1. In order to see this, it is helpful to consider Fig. 3 which depicts the players' best response correspondences in this game. The first triangle should be read as follows. Every point in the triangle represents a probabilistic belief of player 1 about player 2's strategies. The three areas in this triangle represent the sets of beliefs for which the strategies $a, b$ and $c$ are optimal, respectively. Similarly for the second triangle. Consider a Nash equilibrium $\left(\mu_{1}, \mu_{2}\right)$ of the game, where $\mu_{i}$ is a probability distribution over $i$ 's strategies for $i=1,2$. We show that $\mu_{1}$ must assign probability 1 to $b$, and $\mu_{2}$ must assign probability 1 to $e$. Suppose, contrary to what we want to show, that $\mu_{1}$ assigns positive probability to $a$. Then, since $\left(\mu_{1}, \mu_{2}\right)$ is a Nash equilibrium, $a$ must be optimal for player 1 against $\mu_{2}$. By Fig. 2, $\mu_{2}$ must then assign positive probability to $d$, and hence $d$ must be optimal against $\mu_{1}$. By Fig. 2, $\mu_{1}$ must then

\footnotetext{
1 For more than two players, one needs to impose that beliefs about different opponents be stochastically independent in order to obtain the equivalence with rationalizability.
} 

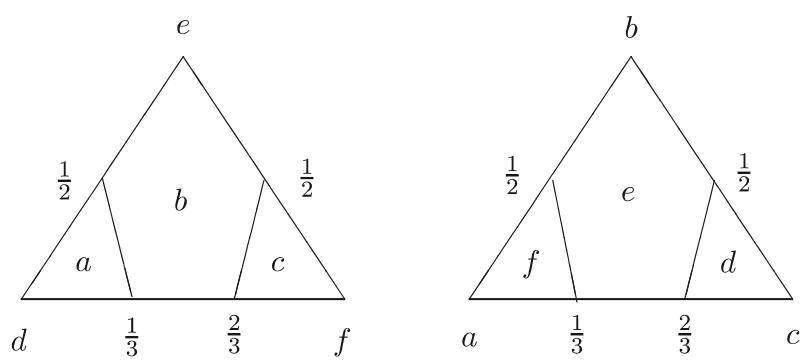

Fig. 3 Best response correspondences for the game in Fig. 2

assign positive probability to $c$, and hence $c$ must be optimal against $\mu_{2}$. So, both $a$ and $c$ should be optimal against $\mu_{2}$. However, from the first triangle it is clear that $a$ and $c$ cannot both be optimal against $\mu_{2}$, and hence we have a contradiction. By symmetry, one can similarly prove that $\mu_{1}$ cannot assign positive probability to $c$, and that $\mu_{2}$ cannot assign positive probability to $d$ or $f$. Hence, the only strategy that can rationally be chosen by player 1 in a Nash equilibrium is $b$, and therefore $b$ is the only Nash strategy for player 1.

Now, let us compare the scenario above which led to the non-Nash strategy $a$ with the simpler scenario that led to the Nash strategy $b$. One fundamental difference between both scenarios is that the first assumes that player 1 believes that player 2 is wrong about 1's belief, whereas the second scenario assumes that player 1 believes that player 2 is right about 1's belief. Namely, in the first scenario player 1 believes that player 2 chooses $d$, but he believes that player 2 believes that he believes that player 2 chooses $f$, and not $d$.

Even stronger, the choice $a$ can only be justified by scenarios in which player 1 believes that player 2 is wrong about 1's belief. In order to see this, assume that strategy $a$ would be optimal for player 1 . Then, player 1 must believe with positive probability that player 2 chooses $d$. In turn, player 2 can only rationally choose $d$ if he believes with positive probability that player 1 chooses $c$. Hence, player 1 must believe with positive probability that player 2 believes with positive probability that player 1 chooses $c$. However, there is no belief for player 1 for which both $a$ and $c$ are rational, and hence player 1 must believe with positive probability that player 2 believes with positive probability that player 1's belief is different from his true belief.

Say that a belief hierarchy $\beta_{i}$ for player $i$ has self-referential beliefs if $\beta_{i}$ believes that every opponent $j$ believes that $i$ 's belief hierarchy is $\beta_{i}$. For the example in Fig. 2 , we may thus conclude that the difference between Nash strategies and rationalizable non-Nash strategies is that the former can be justified by a self-referential belief hierarchy, whereas the latter cannot. The question is whether this is true generally. In Theorem 4.5 we show that this is true for the class of two-player games. More precisely, we show in Theorem 4.5 that in every two-player game, a player who (1) believes in the opponent's rationality (BOR), (2) has self-referential beliefs (SRB), (3) believes that his opponent BOR, and (4) believes that his opponent has SRB, must choose a Nash strategy. 
The four conditions above are no longer enough if we turn to more than two players. Similarly to existing foundations for Nash equilibrium in the literature, we encounter the following two problems for more than two players: (a) We must guarantee that player $i$ 's belief about opponent $j$ 's strategy choice should be stochastically independent from $i$ 's belief about opponent $k$ 's strategy choice, and (b) we must guarantee that player $i$ believes that opponents $j$ and $k$ hold the same belief about player $l$ 's strategy choice. In this paper, we guarantee these events by imposing conditionally independent beliefs and projective beliefs. Conditionally independent beliefs (CIB) is taken from Brandenburger and Friedenberg (2006), and says that for any given profile of belief hierarchies for $i$ 's opponents, player $i$ 's belief about his opponents' strategy choices conditional on this profile of belief hierarchies should be uncorrelated. In other words, any correlation in $i$ 's belief about his opponents' strategy choices should be due to correlation in his belief about the opponents' belief hierarchies. The idea behind this condition is that players with fixed belief hierarchies are assumed to choose their strategies independently. Projective beliefs (PB), in turn, states that player $i$ believes that $j$ 's belief about player $k$ is the same as his own belief about player $k$. That is, player $i$ projects his own belief about player $k$ on player $j$. In Theorem 4.5 we show that for three players or more, a player who (1) BOR, (2) has SRB, (3) believes that his opponents BOR, (4) believes that his opponents have SRB, and, in addition, (5) has $\mathrm{CIB}$, (6) believes that his opponents have $\mathrm{PB}$, and (7) believes that his opponents have CIB, must choose a Nash strategy. At this stage, one may wonder why we did not impose that the player himself has PB. The reason is that this property follows from the assumptions that the player has SRB and believes that every opponent has PB (see Lemma 4.2).

The outline of this paper is as follows: In Sect. 2 we present our epistemic model. Section 3 formally introduces the notions of BOR, SRB, PB and CIB. In Sect. 4 we show that every player who satisfies the conditions (1)-(7) above must choose a Nash strategy. In Theorem 4.7 we prove the converse of this result, namely that every Nash strategy can rationally be chosen by a player who satisfies these conditions (1)-(7). In Sect. 5 we prove that none of these seven conditions can be dropped in Theorem 4.5. In particular, this implies that the seven conditions are logically independent. In Sect. 6 we discuss the intuitive content of Theorems 4.5 and 4.7, with a special focus on SRB and PB. In Sect. 7 we compare our conditions with sufficient conditions for Nash equilibrium as provided in Aumann and Brandenburger (1995), Asheim (2006) and Brandenburger and Dekel (1987).

\section{Epistemic model}

Let $I$ be a finite set of players. A finite game is a tuple $\Gamma=\left(S_{i}, u_{i}\right)_{i \in I}$, where $S_{i}$ is the finite set of strategies for player $i$, and $u_{i}: \times_{j \in I} S_{j} \rightarrow \mathbb{R}$ is player $i$ 's utility function. We shall assume throughout that a player believes that the utility functions are as specified by $\Gamma$, that a player believes that all players believe this, and so on. For every finite set $X$, let $\Delta(X)$ denote the set of probability distributions on $X$.

Definition 2.1 (Epistemic model) A finite epistemic model for the game $\Gamma$ is a tuple

$$
\mathcal{M}=\left(T_{i}, \beta_{i}\right)_{i \in I}
$$


where, for every player $i, T_{i}$ is a finite set of types, and $\beta_{i}$ is a one-to-one function from $T_{i}$ to $\Delta\left(S_{-i} \times T_{-i}\right)$.

Here, $S_{-i}$ is a short way to write $\times_{j \neq i} S_{j}$, and similarly for $T_{-i}$. The interpretation of $\beta_{i}$ is that for every type $t_{i} \in T_{i}$, the image $\beta_{i}\left(t_{i}\right)$ denotes $t_{i}$ 's probabilistic belief about the opponents' strategy choices and types. For every event $E \subseteq S_{-i} \times T_{-i}$ for player $i$ and every number $p \in[0,1]$, we say that type $t_{i}$ believes the event $E$ with probability $p$ if $\beta_{i}\left(t_{i}\right)(E)=p$. We say that $t_{i}$ believes $E$ if $\beta_{i}\left(t_{i}\right)(E)=1$. For instance, we say that $t_{i}$ believes that player $j$ has type $t_{j}$ if $t_{i}$ assigns probability 1 to the set of strategy-type profiles in $S_{-i} \times T_{-i}$ where player $j$ 's type is $t_{j}$.

\section{Restrictions on beliefs}

In this section we discuss four conditions that one may impose on a player's beliefs: belief in the opponents' rationality, self-referential beliefs, projective beliefs and conditionally independent beliefs. We first need some additional terminology. For every type $t_{i}$ and strategy profile $s_{-i} \in S_{-i}$, we denote by $\beta_{i}\left(t_{i}\right)\left(s_{-i}\right)$ the probability that the belief $\beta_{i}\left(t_{i}\right)$ assigns to the set $\left\{s_{-i}\right\} \times T_{-i}$. For every strategy $s_{i} \in S_{i}$, we denote by

$$
u_{i}\left(s_{i}, t_{i}\right):=\sum_{s_{-i} \in S_{-i}} \beta_{i}\left(t_{i}\right)\left(s_{-i}\right) u_{i}\left(s_{i}, s_{-i}\right)
$$

the expected utility for type $t_{i}$ of choosing strategy $s_{i}$. We say that strategy $s_{i}$ is rational for type $t_{i}$ if $u_{i}\left(s_{i}, t_{i}\right) \geq u_{i}\left(s_{i}^{\prime}, t_{i}\right)$ for every $s_{i}^{\prime} \in S_{i}$.

Definition 3.1 (Belief in the opponents' rationality) Type $t_{i}$ is said to believe in the opponents' rationality if for every opponent $j$, and every strategy-type pair $\left(s_{j}, t_{j}\right) \in$ $S_{j} \times T_{j}$ to which $t_{i}$ assigns positive probability, the strategy $s_{j}$ is rational for type $t_{j}$.

Definition 3.2 (Self-referential beliefs) Type $t_{i}$ is said to have self-referential beliefs if for every $p \in[0,1]$ and every event $E \subseteq S_{-i} \times T_{-i}$ which $t_{i}$ believes with probability $p$, type $t_{i}$ believes that all opponents believe that player $i$ believes $E$ with probability $p$.

Hence, a player with self-referential beliefs thinks that his opponents hold correct beliefs about his own beliefs. Now, let $j$ be an opponent of $i$, and let $E_{j} \subseteq S_{j} \times T_{j}$ be an event about player $j$. We say that $t_{i}$ believes $E_{j}$ with probability $p$ if $t_{i}$ assigns probability $p$ to the event

$$
E_{j} \times\left(\times_{k \neq i, j}\left(S_{k} \times T_{k}\right)\right) .
$$

Definition 3.3 (Projective beliefs) Type $t_{i}$ is said to have projective beliefs if for every pair of opponents $j, k$ and every event $E_{k} \subseteq S_{k} \times T_{k}$ about player $k$ : if $t_{i}$ believes $E_{k}$ with probability $p$, then $t_{i}$ believes that $j$ believes $E_{k}$ with probability $p$.

Intuitively, a player with projective beliefs projects his belief about an opponent on his other opponents. Of course, this condition only imposes restrictions if there are at least three players. Our last condition, conditionally independent beliefs, is taken from Brandenburger and Friedenberg (2006). It states that for every given profile $t_{-i}$ of 
opponents' types which is deemed possible by type $t_{i}$, his belief about the opponents' strategies conditional on $t_{-i}$ should be uncorrelated. In other words, any correlation in $t_{i}$ 's belief about the opponents' strategy choices should come from correlation in his belief about the opponents' types. The idea behind this condition is that types are assumed to choose their strategies independently since pre-play communication is not allowed. However, player $i$ 's belief about $j$ 's choice may still be dependent on his belief about $k$ 's choice if his belief about $j$ 's type is dependent on his belief about $k$ 's type. To formalize this condition, we need some terminology. Let $t_{-i}$ be a profile of opponents' types to which $t_{i}$ assigns positive probability, and let $s_{-i}$ be a profile of opponents' strategies. By

$$
\beta_{i}\left(t_{i}\right)\left(s_{-i} \mid t_{-i}\right):=\frac{\beta_{i}\left(t_{i}\right)\left(s_{-i}, t_{-i}\right)}{\beta_{i}\left(t_{i}\right)\left(t_{-i}\right)}
$$

we denote the probability that $t_{i}$ assigns to the strategy profile $s_{-i}$, conditional on the event that the opponents' types are given by $t_{-i}$. Here, $\beta_{i}\left(t_{i}\right)\left(t_{-i}\right)$ denotes the probability that $t_{i}$ assigns to the event that the opponents' types are $t_{-i}$. Similarly, for every opponent $j$, every type $t_{j}$ to which $t_{i}$ assigns positive probability, and every strategy $s_{j} \in S_{j}$, we denote by

$$
\beta_{i}\left(t_{i}\right)\left(s_{j} \mid t_{j}\right):=\frac{\beta_{i}\left(t_{i}\right)\left(s_{j}, t_{j}\right)}{\beta_{i}\left(t_{i}\right)\left(t_{j}\right)}
$$

the probability that $t_{i}$ assigns to strategy choice $s_{j}$, conditional on the event that $j$ 's type is $t_{j}$. Here, $\beta_{i}\left(t_{i}\right)\left(s_{j}, t_{j}\right)$ denotes the probability that $t_{i}$ assigns to the event that $j$ chooses $s_{j}$ and has type $t_{j}$, whereas $\beta_{i}\left(t_{i}\right)\left(t_{j}\right)$ is the probability that $t_{i}$ assigns to the event that $j$ has type $t_{j}$.

Definition 3.4 (Conditionally independent beliefs) Type $t_{i}$ is said to have conditionally independent beliefs if for every $t_{-i} \in T_{-i}$ with $\beta_{i}\left(t_{i}\right)\left(t_{-i}\right)>0$, and every $s_{-i} \in$ $S_{-i}$ :

$$
\beta_{i}\left(t_{i}\right)\left(s_{-i} \mid t_{-i}\right)=\prod_{j \neq i} \beta_{i}\left(t_{i}\right)\left(s_{j} \mid t_{j}\right)
$$

In fact, this condition combines the restrictions of conditional independence and sufficiency in Brandenburger and Friedenberg (2006). In their model, sufficiency states that player $i$ 's belief about player $j$ 's strategy choice should be independent of his belief about some other opponent's type. Obviously, our notion of conditionally independent beliefs satisfies this additional requirement. It should be clear that conditionally independent beliefs only imposes restrictions if there are at least three players.

As an abbreviation, we denote by BOR, SRB, PB and CIB the events that types believe in the opponents' rationality, have self-referential beliefs, have projective beliefs, and have conditionally independent beliefs, respectively. We say that type $t_{i}$ believes that every opponent satisfies BOR if $\beta_{i}\left(t_{i}\right)$ only assigns positive probability to opponents' types that satisfy BOR. Similarly, we define the events that $t_{i}$ believes that every opponent satisfies SRB, PB and CIB. 


\section{Relation with Nash strategies}

In this section we show that every type which satisfies BOR, SRB, and CIB, believes that every opponent satisfies BOR, SRB, PB and CIB, and chooses rationally, must choose a Nash strategy. A profile $\left(\mu_{i}\right)_{i \in I}$ of probability distributions $\mu_{i} \in \Delta\left(S_{i}\right)$ is called a Nash equilibrium for the game $\Gamma$ if, for every player $i, \mu_{i}\left(s_{i}\right)>0$ only if $u_{i}\left(s_{i}, \mu_{-i}\right) \geq u_{i}\left(s_{i}^{\prime}, \mu_{-i}\right)$ for every $s_{i}^{\prime} \in S_{i}$. Here,

$$
u_{i}\left(s_{i}, \mu_{-i}\right):=\sum_{s_{-i} \in S_{-i}} \prod_{j \neq i} \mu_{j}\left(s_{j}\right) u_{i}\left(s_{i}, s_{-i}\right)
$$

denotes the expected utility for player $i$ of choosing $s_{i}$ if his belief about the opponents' strategies is given by $\mu_{-i}$.

We call a strategy $s_{i}$ a Nash strategy for $\Gamma$ if there is a Nash equilibrium $\left(\mu_{i}\right)_{i \in I}$ for $\Gamma$ such that $u_{i}\left(s_{i}, \mu_{-i}\right) \geq u_{i}\left(s_{i}^{\prime}, \mu_{-i}\right)$ for every $s_{i}^{\prime} \in S_{i}$. Since this definition is nonstandard, it requires some further explanation. In many models, a Nash equilibrium $\left(\mu_{i}\right)_{i \in I}$ is interpreted as an equilibrium in beliefs, and $\mu_{-i}$ represents player $i$ 's belief about his opponents' strategy choices. This is also the viewpoint I take in this paper. With this interpretation in mind, one can ask the following natural question: What are the behavioral consequences of requiring that player $i$ 's belief about the opponents' strategy choices is part of a Nash equilibrium in beliefs? In other words, which strategies can player $i$ rationally choose if his belief $\mu_{-i}$ about the opponents' strategies is part of a Nash equilibrium in beliefs? The answer must then be: any Nash strategy as defined above. Hence, the definition of a Nash strategy formalizes the behavioral consequences of assuming that player $i$ 's beliefs about the opponents is part of a Nash equilibrium in beliefs. Recall from the introduction that every strategy that is assigned positive probability in some Nash equilibrium in beliefs is also a Nash strategy, but the converse is not true.

Before we prove our theorem on the relation with Nash strategies, we first derive some implications of SRB, PB, CIB, and belief in these events. In each of these lemmas, we assume that $\mathcal{M}=\left(T_{i}, \beta_{i}\right)_{i \in I}$ is a finite epistemic model for a finite game $\Gamma$.

Lemma 4.1 Let $t_{i} \in T_{i}$ be a type with SRB. Then, $t_{i}$ believes that every opponent believes that $i$ 's type is $t_{i}$.

Proof Choose an arbitrary event $E \subseteq S_{-i} \times T_{-i}$ for player $i$, and assume that $t_{i}$ believes $E$ with probability $p$. By SRB, $t_{i}$ believes that every opponent believes that $i$ believes $E$ with probability $p$. Since this holds for every $E$, and since the function $\beta_{i}: T_{i} \rightarrow \Delta\left(S_{-i} \times T_{-i}\right)$ in the epistemic model is one-to-one, $t_{i}$ believes that every opponent believes that $i$ 's type is $t_{i}$.

Lemma 4.2 Let $t_{i} \in T_{i}$ be a type with $S R B$ which believes that every opponent has $P B$. Then, $t_{i}$ has $P B$.

Proof Suppose that $j$ and $k$ are two different opponents of player $i$. Let $E_{k} \subseteq S_{k} \times T_{k}$ be an event which $t_{i}$ believes with probability $p$, and let $t_{j} \in T_{j}$ be a type to which $t_{i}$ assigns positive probability. We show that $t_{j}$ believes $E_{k}$ with probability $p$ as well. 
Since $t_{i}$ has SRB, we know from Lemma 4.1 that $t_{j}$ believes that $i$ 's type is $t_{i}$. Since $t_{i}$ believes that $j$ has $\mathrm{PB}$, it must be the case that $t_{j}$ has $\mathrm{PB}$, and hence $t_{j}$ 's belief about player $k$ must be the same as $t_{i}$ 's belief about player $k$. Consequently, $t_{j}$ must believe $E_{k}$ with probability $p$. We may thus conclude that $t_{i}$ has PB.

Lemma 4.3 Let $t_{i}$ be a type with SRB which believes that every opponent has $P B$ and $S R B$. Then, there are opponents' types $t_{k} \in T_{k}, k \in I \backslash\{i\}$, such that (1) $t_{i}$ believes for every $j \neq i$ that $j$ 's type is $t_{j}$, (2) for every $j \neq i$, type $t_{j}$ believes that $i$ 's type is $t_{i}$, and (3) for every $j, k \neq i$, type $t_{j}$ believes that $k$ 's type is $t_{k}$.

Proof Suppose that $j \neq i$, and that $t_{i}$ assigns positive probability to type $t_{j} \in T_{j}$. By Lemma 4.1 we know that $t_{i}$ believes that $j$ believes that $i$ 's type is $t_{i}$. Hence, $t_{j}$ must believe that $i$ 's type is $t_{i}$. Since $t_{i}$ believes that $j$ has SRB, type $t_{j}$ must have SRB. By applying Lemma 4.1 to $t_{j}$, we may conclude that $t_{j}$ believes that $i$ believes that $j$ 's type is $t_{j}$. Since we have seen that $t_{j}$ believes that $i$ 's type is $t_{i}$, it follows that $t_{i}$ must believe that $j$ 's type is $t_{j}$. Hence, we have shown properties (1) and (2). It remains to show (3). By Lemma 4.2 we know that $t_{i}$ has PB. Suppose that $j, k \neq i$. Since $t_{i}$ believes that $k$ 's type is $t_{k}$, and since $t_{i}$ has $\mathrm{PB}$, it follows that $t_{i}$ believes that $j$ believes that $k$ 's type is $t_{k}$. Since $t_{i}$ believes that $j$ 's type is $t_{j}$, type $t_{j}$ believes that $k$ 's type is $t_{k}$. This completes the proof.

Lemma 4.4 Let $t_{i} \in T_{i}$ be a type that has $S R B$ and CIB, and believes that every opponent has $S R B, P B$ and $C I B$. Then, there are probability distributions $\mu_{k} \in \Delta\left(S_{k}\right)$, $k \in I$, such that (1) $t_{i}$ believes that every opponents' strategy profile $\left(s_{j}\right)_{j \neq i}$ is chosen with probability $\prod_{j \neq i} \mu_{j}\left(s_{j}\right)$, (2) for every $j \neq i$, type $t_{i}$ believes that $j$ believes that every opponents' strategy profile $\left(s_{k}\right)_{k \neq j}$ is chosen with probability $\prod_{k \neq j} \mu_{k}\left(s_{k}\right)$, and (3) for every $j \neq i$, type $t_{i}$ believes that $j$ believes that $i$ believes that every opponents' strategy profile $\left(s_{j}\right)_{j \neq i}$ is chosen with probability $\prod_{j \neq i} \mu_{j}\left(s_{j}\right)$.

Proof By Lemma 4.3, there are opponents' types $t_{k} \in T_{k}, k \in I \backslash\{i\}$, such that (1) $t_{i}$ believes for every $j \neq i$ that $j$ 's type is $t_{j}$, (2) for every $j \neq i$, type $t_{j}$ believes that $i$ 's type is $t_{i}$, and (3) for every $j, k \neq i$, type $t_{j}$ believes that $k$ 's type is $t_{k}$. Let $t_{-i}:=\left(t_{j}\right)_{j \neq i}$. Since $t_{i}$ has CIB, it holds that

$$
\beta_{i}\left(t_{i}\right)\left(s_{-i} \mid t_{-i}\right)=\prod_{j \neq i} \beta_{i}\left(t_{i}\right)\left(s_{j} \mid t_{j}\right)
$$

for every $s_{-i} \in S_{-i}$. Now, define $\mu_{j}\left(s_{j}\right):=\beta_{i}\left(t_{i}\right)\left(s_{j} \mid t_{j}\right)$ for every $j \neq i$ and every $s_{j} \in S_{j}$. Then, by (4.1) and the fact that $t_{i}$ believes that every opponent $j$ has type $t_{j}$, type $t_{i}$ believes that every opponents' strategy profile $s_{-i}$ is chosen with probability $\prod_{j \neq i} \mu_{j}\left(s_{j}\right)$. We have thus shown property (1) of this lemma.

Now, choose some fixed opponent $j \neq i$. Since $t_{i}$ believes that $j$ has CIB, and $t_{i}$ believes that $j$ 's type is $t_{j}$, type $t_{j}$ has CIB. Hence,

$$
\beta_{j}\left(t_{j}\right)\left(s_{-j} \mid t_{-j}\right)=\prod_{k \neq j} \beta_{j}\left(t_{j}\right)\left(s_{k} \mid t_{k}\right)
$$

for every opponents' strategy profile $s_{-j}$. Choose some arbitrary player $k \notin\{i, j\}$. Since, by Lemma 4.2, $t_{i}$ has PB and believes that $j$ 's type is $t_{j}$, type $t_{i}$ 's belief 
about $k$ 's strategy choice must be equal to $t_{j}$ 's belief about $k$ 's strategy choice, and hence $\beta_{j}\left(t_{j}\right)\left(s_{k} \mid t_{k}\right)=\beta_{i}\left(t_{i}\right)\left(s_{k} \mid t_{k}\right)=\mu_{k}\left(s_{k}\right)$ for every $s_{k} \in S_{k}$. Define $\mu_{i}\left(s_{i}\right):=$ $\beta_{j}\left(t_{j}\right)\left(s_{i} \mid t_{i}\right)$ for every $s_{i} \in S_{i}$. Together with (4.2), we may then conclude that $t_{j}$ believes that every opponents' strategy profile $s_{-j}$ is chosen with probability $\prod_{k \neq j} \mu_{k}\left(s_{k}\right)$. So, $t_{i}$ believes that $j$ believes that every opponents' strategy profile $s_{-j}$ is chosen with probability $\prod_{k \neq j} \mu_{k}\left(s_{k}\right)$.

Finally, choose some arbitrary player $k \notin\{i, j\}$. Since $t_{i}$ believes that $k$ has CIB, and believes that $k$ 's type is $t_{k}$, type $t_{k}$ must have CIB. Hence,

$$
\beta_{k}\left(t_{k}\right)\left(s_{-k} \mid t_{-k}\right)=\prod_{l \neq k} \beta_{k}\left(t_{k}\right)\left(s_{l} \mid t_{l}\right)
$$

for every $s_{-k} \in S_{-k}$. Let $l \notin\{i, k\}$. By Lemma 4.2 we know that $t_{i}$ has PB. Since $t_{i}$ has $\mathrm{PB}$, and believes that $k$ 's type is $t_{k}$, type $t_{i}$ 's belief about $l$ 's strategy choice must be equal to $k$ 's belief about $l$ 's strategy choice, and hence $\beta_{k}\left(t_{k}\right)\left(s_{l} \mid t_{l}\right)=\beta_{i}\left(t_{i}\right)\left(s_{l} \mid t_{l}\right)=$ $\mu_{l}\left(s_{l}\right)$ for every $s_{l} \in S_{l}$. Since $t_{i}$ believes that $j$ has PB, and believes that $j$ has type $t_{j}$, type $t_{j}$ must have PB. Hence, $t_{j}$ 's belief about $i$ 's strategy choice must be equal to $k$ 's belief about $i$ 's strategy choice, which implies that $\beta_{k}\left(t_{k}\right)\left(s_{i} \mid t_{i}\right)=\beta_{j}\left(t_{j}\right)\left(s_{i} \mid t_{i}\right)=$ $\mu_{i}\left(s_{i}\right)$. Combined with (4.3) we obtain that $t_{k}$ believes that every opponents' strategy profile $s_{-k}$ is chosen with probability $\prod_{l \neq k} \mu_{l}\left(s_{l}\right)$. So, $t_{i}$ believes that every $k \neq j$ believes that every opponents' strategy profile $s_{-k}$ is chosen with probability $\prod_{l \neq k} \mu_{l}\left(s_{l}\right)$. Hence, we have shown property (2).

Since we already know from Lemma 4.1 that $t_{i}$ believes that every opponent $j$ believes that $i$ 's type is $t_{i}$, property (3) follows immediately. This completes the proof.

We are now ready to prove our main theorem.

Theorem 4.5 Let $\mathcal{M}=\left(T_{i}, \beta_{i}\right)_{i \in I}$ be a finite epistemic model for a finite game $\Gamma$. Let $t_{i} \in T_{i}$ be a type that satisfies BOR, SRB and CIB, and believes that every opponent satisfies BOR, SRB, PB and CIB. Then, every strategy that is rational for $t_{i}$ is a Nash strategy for $\Gamma$.

Proof Let $t_{i}$ be a type that satisfies BOR, SRB and CIB, and believes that every opponent satisfies BOR, SRB, PB and CIB. Let $\mu_{k} \in \Delta\left(S_{k}\right), k \in I$, be the probability distributions obtained from Lemma 4.4. We show that $\left(\mu_{k}\right)_{k \in I}$ is a Nash equilibrium.

Suppose first that $j \neq i$ and that $\mu_{j}\left(s_{j}\right)>0$ for some $s_{j} \in S_{j}$. Since $t_{i}$ believes in $j$ 's rationality, and, by (2), believes that $j$ 's belief about the opponents' strategy choices is given by $\mu_{-j}=\left(\mu_{k}\right)_{k \neq j}$, it follows that $s_{j}$ must be optimal against $\mu_{-j}$. Finally, let $\mu_{i}\left(s_{i}\right)>0$ for some $s_{i} \in S_{i}$. Choose some arbitrary opponent $j$. Since, by (2), $t_{i}$ 's belief about $j$ 's belief about the opponents' strategy choice is given by $\mu_{-j}$, type $t_{i}$ believes that $j$ believes that $i$ chooses $s_{i}$ with positive probability. Since $t_{i}$ believes that $j$ believes in $i$ 's rationality, and since, by (3), $t_{i}$ 's belief about $j$ 's belief about $i$ 's belief about the opponents' strategy choices is given by $\mu_{-i}$, it follows that $s_{i}$ must be optimal against $\mu_{-i}$. Hence, $\left(\mu_{j}\right)_{j \in I}$ is a Nash equilibrium.

Now, choose some strategy $s_{i}$ that is rational for $t_{i}$. Since, by (1), $t_{i}$ 's belief about the opponents' strategy choices is $\mu_{-i}$, strategy $s_{i}$ must be optimal against $\mu_{-i}$. As $\left(\mu_{i}, \mu_{-i}\right)$ is a Nash equilibrium, $s_{i}$ is a Nash strategy. This completes the proof. 
Theorem 4.5 thus provides sufficient doxastic conditions for choosing Nash strategies. The question remains whether the conditions in this theorem also lead, in some sense, to Nash equilibrium in beliefs. The answer is "yes." Consider, namely, a type $t_{i}$ that satisfies BOR, SRB and CIB, and believes that every opponent satisfies BOR, $\mathrm{SRB}, \mathrm{PB}$ and CIB. In the proof of Theorem 4.5, we have shown that there exist probability distributions $\mu_{k} \in \Delta\left(S_{k}\right), k \in I$, such that (1) for $j \neq i, \mu_{j}$ is $t_{i}$ 's belief about $j$ 's strategy choice, and also $t_{i}$ 's belief about any other player's belief about player $j$ 's strategy choice, and (2) $\mu_{i}$ is $t_{i}$ 's belief about any other player's belief about his own strategy choice. Moreover, we have shown in the proof that the profile $\left(\mu_{k}\right)_{k \in I}$ is a Nash equilibrium in beliefs. We thus obtain the following corollary.

Corollary 4.6 Let $\mathcal{M}=\left(T_{i}, \beta_{i}\right)_{i \in I}$ be a finite epistemic model for a finite game $\Gamma$. Let $t_{i} \in T_{i}$ be a type that satisfies BOR, SRB and CIB, and believes that every opponent satisfies BOR, SRB, PB and CIB. For every player $j \neq i$, let $\mu_{j} \in \Delta\left(S_{j}\right)$ be $t_{i}$ 's belief about $j$ 's strategy choice, and let $\mu_{i}$ be $t_{i}$ 's belief about any other player's belief about his own strategy choice. Then, $\left(\mu_{j}\right)_{j \in I}$ is a Nash equilibrium in beliefs.

Our last result shows that every Nash strategy can be chosen rationally by a type that satisfies BOR, SRB and CIB, and believes that his opponents satisfy BOR, SRB, PB and CIB. In particular, the combination of these seven events is shown to be possible.

Theorem 4.7 Let $\Gamma$ be a finite game, and let $s_{i}$ be a Nash strategy for player $i$ in $\Gamma$. Then, there exists a finite epistemic model $\mathcal{M}=\left(T_{i}, \beta_{i}\right)_{i \in I}$ for $\Gamma$, and a type $t_{i} \in T_{i}$, such that $s_{i}$ is rational for $t_{i}$, type $t_{i}$ satisfies BOR, SRB, and CIB, and believes that every opponent satisfies BOR, SRB, $P B$ and $C I B$.

Proof Let $s_{i}$ be a Nash strategy for $\Gamma$. Then, there is a Nash equilibrium $\left(\mu_{j}\right)_{j \in I}$ for $\Gamma$ such that $s_{i}$ is optimal for $i$ against $\mu_{-i}$. We define the epistemic model $\mathcal{M}=$ $\left(T_{i}, \beta_{i}\right)_{i \in I}$ as follows: Define $T_{j}:=\left\{\hat{t}_{j}\right\}$ for every player $j$, and let $\beta_{j}\left(\hat{t}_{j}\right)$ be the probability distribution on $S_{-j} \times T_{-j}$ given by

$$
\beta_{j}\left(\hat{t}_{j}\right)\left(s_{-j}, t_{-j}\right):=\left\{\begin{aligned}
\prod_{k \neq j} \mu_{k}\left(s_{k}\right), & \text { if } t_{-j}=\hat{t}_{-j} \\
0, & \text { otherwise. }
\end{aligned}\right.
$$

We show that for every player $j$, the type $\hat{t}_{j}$ satisfies BOR, SRB, PB and CIB. Since $\hat{t}_{i}$ believes, for every opponent $j$, that $j$ 's type is $\hat{t}_{j}$, it would follow that $\hat{t}_{i}$ believes that every opponent satisfies these four conditions as well.

BOR: Let $k$ be an opponent for $j$, and let $s_{k}$ be a strategy for $k$ with $\beta_{j}\left(\hat{t}_{j}\right)\left(s_{k}, \hat{t}_{k}\right)>0$. Then, $\mu_{k}\left(s_{k}\right)>0$. Since $\left(\mu_{j}\right)_{j \in I}$ is a Nash equilibrium, $s_{k}$ is optimal for $k$ against $\mu_{-k}$, and hence $s_{k}$ is rational for $\hat{t}_{k}$. Therefore, $\hat{t}_{j}$ satisfies BOR.

$S R B$ : By construction, $\hat{t}_{j}$ believes that every opponent $k$ has type $\hat{t}_{k}$. Moreover, every such type $\hat{t}_{k}$ believes that $j$ 's type is $\hat{t}_{j}$. Hence, $\hat{t}_{j}$ believes that every opponent believes that $j$ 's type is $\hat{t}_{j}$. Now, suppose that $\hat{t}_{j}$ believes an event $E$ with probability $p$. Then, since $\hat{t}_{j}$ believes that every opponent believes that $j$ 's type is $\hat{t}_{j}$, type $\hat{t}_{j}$ believes that every opponent believes that $j$ believes $E$ with probability $p$. Consequently, $\hat{t}_{j}$ satisfies SRB. 
$P B$ : Let $k, l$ be two different opponents for $j$, let $E_{k} \subseteq S_{k} \times T_{k}$, and suppose that $\hat{t}_{j}$ believes $E_{k}$ with probability $p$. By construction, $\hat{t}_{j}$ believes that $l$ 's type is $\hat{t}_{l}$, and $\hat{t}_{l}$ 's belief about player $k$ is the same as $\hat{t}_{j}$ 's belief about player $k$. Hence, $\hat{t}_{j}$ believes that $l$ believes $E_{k}$ with probability $p$. We may thus conclude that $\hat{t}_{j}$ satisfies PB.

$C I B$ : It follows immediately from the construction of the epistemic model that $\hat{t}_{j}$ satisfies CIB.

Hence, we may conclude that every $\hat{t}_{j}$ satisfies BOR, SRB, PB and CIB. This implies that $\hat{t}_{i}$ satisfies these four conditions, and believes that every opponent satisfies these four conditions too. Recall that the Nash equilibrium $\left(\mu_{j}\right)_{j \in I}$ was chosen such that $s_{i}$ is optimal for $i$ against $\mu_{-i}$. Since $\mu_{-i}$ is $\hat{t}_{i}$ 's belief about the opponents' strategy choices, $s_{i}$ is rational for $\hat{t}_{i}$. This completes the proof.

\section{No conditions can be dropped}

In Theorem 4.5 we have shown that the conditions BOR, SRB, CIB, and belief in BOR, SRB, PB and CIB, lead to Nash strategy choices. So, in total we have seven conditions that we impose on a player's beliefs. In this section we prove that this result no longer holds if we drop one of these seven conditions. In particular, we show that these seven conditions are logically independent.

Dropping BOR: Consider the two-player game in Fig. 4, where player 1 chooses the rows and player 2 the columns. Construct an epistemic model $\mathcal{M}$ such that $T_{1}=\left\{t_{1}\right\}$, $T_{2}=\left\{t_{2}\right\}, \beta_{1}\left(t_{1}\right)$ assigns probability 1 to $\left(d, t_{2}\right)$, and $\beta_{2}\left(t_{2}\right)$ assigns probability 1 to $\left(a, t_{1}\right)$. Then, $t_{1}$ does not BOR, since $d$ is not optimal for $t_{2}$. On the other hand, $t_{1}$ believes that player $2 \mathrm{BOR}$, since $a$ is optimal for $t_{1}$. Type $t_{1}$ has SRB since $t_{1}$ believes that player 2 believes that his type is $t_{1}$. Similarly, $t_{2}$ has SRB, and hence $t_{1}$ believes in SRB. Clearly, $t_{1}$ has CIB, and believes that player 2 has PB and CIB, since these conditions are automatically satisfied for two players. Hence, $t_{1}$ does not BOR, but satisfies the other six conditions. Strategy $a$ is rational for $t_{1}$, but $a$ is not a Nash strategy.

Dropping belief in BOR: Consider again the two-player game in Fig. 4. Construct an epistemic model $\mathcal{M}$ such that $T_{1}=\left\{t_{1}\right\}, T_{2}=\left\{t_{2}\right\}, \beta_{1}\left(t_{1}\right)$ assigns probability 1 to $\left(d, t_{2}\right)$, and $\beta_{2}\left(t_{2}\right)$ assigns probability 1 to $\left(c, t_{1}\right)$. Then, $t_{1}$ does not believe in BOR, since $c$ is not optimal for $t_{1}$. However, $t_{1}$ satisfies the other six conditions. Strategy $a$ is optimal for $t_{1}$, but $a$ is not a Nash strategy.

\begin{tabular}{|l|l|l|}
\hline & $d$ & $e$ \\
\hline$a$ & 2,0 & 1,1 \\
\hline$b$ & 1,0 & 2,1 \\
\hline$c$ & 0,1 & 0,0 \\
\hline
\end{tabular}

Fig. 4 BOR and belief in BOR cannot be dropped 


\begin{tabular}{|l|l|l|l|}
\hline & $d$ & $e$ & $f$ \\
\hline$a$ & 3,0 & 0,3 & 0,0 \\
\hline$b$ & 0,3 & 3,0 & 0,0 \\
\hline$c$ & 2,0 & 2,0 & 2,2 \\
\hline
\end{tabular}

Fig. 5 SRB and belief in SRB cannot be dropped

\begin{tabular}{|l|l|l|l|}
\hline$g$ & $d$ & $e$ & $f$ \\
\hline$a$ & $3,3,0$ & $3,0,3$ & $0,2,0$ \\
\hline$b$ & $0,0,0$ & $0,0,0$ & $0,2,0$ \\
\hline$c$ & $2,0,0$ & $2,0,0$ & $2,2,0$ \\
\hline
\end{tabular}

\begin{tabular}{|l|l|l|l|}
\hline$h$ & $d$ & $e$ & $f$ \\
\hline$a$ & $0,0,0$ & $0,3,3$ & $0,2,0$ \\
\hline$b$ & $0,0,0$ & $3,3,0$ & $0,2,0$ \\
\hline$c$ & $2,0,0$ & $2,0,0$ & $2,2,0$ \\
\hline
\end{tabular}

\begin{tabular}{|l|l|l|l|}
\hline$i$ & $d$ & $e$ & $f$ \\
\hline$a$ & $0,0,2$ & $0,0,2$ & $0,2,2$ \\
\hline$b$ & $0,0,2$ & $0,0,2$ & $0,2,2$ \\
\hline$c$ & $2,0,2$ & $2,0,2$ & $2,2,2$ \\
\hline
\end{tabular}

Fig. 6 Belief in PB cannot be dropped

Dropping SRB: Consider the two-player game in Fig. 5. It can be shown that $(c, f)$ is the only Nash equilibrium, and hence $c$ is the only Nash strategy for player 1 . Construct an epistemic model $\mathcal{M}$ such that $T_{1}=\left\{t_{1}^{a}, t_{1}^{b}\right\}, T_{2}=\left\{t_{2}\right\}, \beta_{1}\left(t_{1}^{a}\right)$ assigns probability 1 to $\left(d, t_{2}\right), \beta_{1}\left(t_{1}^{b}\right)$ assigns probability 1 to $\left(e, t_{2}\right)$, and $\beta_{2}\left(t_{2}\right)$ assigns probability $1 / 2$ to $\left(a, t_{1}^{a}\right)$ and probability $1 / 2$ to $\left(b, t_{1}^{b}\right)$. Then, $t_{1}^{a}$ does not have SRB, since $t_{1}^{a}$ believes that player 2 believes with probability $1 / 2$ that his type is $t_{1}^{b}$ and not $t_{1}^{a}$. However, $t_{1}^{a}$ satisfies the other six conditions. Strategy $a$ is optimal for $t_{1}^{a}$, but $a$ is not a Nash strategy.

Dropping belief in SRB: Consider again the two-player game in Fig. 5. Recall that $(c, f)$ is the only Nash equilibrium, and hence $f$ is the only Nash strategy for player 2. Construct an epistemic model $\mathcal{M}$ such that $T_{1}=\left\{t_{1}^{a}, t_{1}^{b}\right\}, T_{2}=\left\{t_{2}\right\}, \beta_{1}\left(t_{1}^{a}\right)$ assigns probability 1 to $\left(d, t_{2}\right), \beta_{1}\left(t_{1}^{b}\right)$ assigns probability 1 to $\left(e, t_{2}\right)$, and $\beta_{2}\left(t_{2}\right)$ assigns probability $1 / 2$ to $\left(a, t_{1}^{a}\right)$ and probability $1 / 2$ to $\left(b, t_{1}^{b}\right)$. Then, $t_{2}$ does not believe in SRB, since $t_{1}^{a}$ and $t_{1}^{b}$ do not have SRB. However, $t_{2}$ satisfies the other six conditions. Strategy $d$ is optimal for $t_{2}$, but $d$ is not a Nash strategy.

Dropping belief in PB: Consider the three-player game in Fig. 6. Here, player 1 chooses the row, player 2 chooses the column, and player 3 chooses the matrix $(g, h$ or $i)$. Construct an epistemic model $\mathcal{M}$ such that $T_{1}=\left\{t_{1}\right\}, T_{2}=\left\{t_{2}\right\}, T_{3}=$ $\left\{t_{3}\right\}, \beta_{1}\left(t_{1}\right)$ assigns probability 1 to $\left(\left(e, t_{2}\right),\left(g, t_{3}\right)\right), \beta_{2}\left(t_{2}\right)$ assigns probability 1 to $\left(\left(a, t_{1}\right),\left(h, t_{3}\right)\right)$, and $\beta_{3}\left(t_{3}\right)$ assigns probability 1 to $\left(\left(a, t_{1}\right),\left(e, t_{2}\right)\right)$. Type $t_{3}$ does not believe in PB, since $t_{1}$ does not have PB. In order to see this, note that $t_{1}$ believes that player 3 chooses $g$, whereas $t_{1}$ believes that player 2 believes that player 3 chooses $h$. It can easily be verified that $t_{3}$ satisfies the other six conditions.

Strategy $g$ is rational for type $t_{3}$. However, we will show that $g$ is not a Nash strategy. Suppose, contrary to what we want to show, that $g$ would be a Nash strategy. Then, there would be a Nash equilibrium $\left(\mu_{1}, \mu_{2}, \mu_{3}\right)$ such that $g$ would be optimal against $\left(\mu_{1}, \mu_{2}\right)$. Strategy $g$ can only be optimal against $\left(\mu_{1}, \mu_{2}\right)$ if $\mu_{1}(a)>0$ and $\mu_{2}(e)>0$. Since $\left(\mu_{1}, \mu_{2}, \mu_{3}\right)$ is a Nash equilibrium, this implies that $a$ is optimal against $\left(\mu_{2}, \mu_{3}\right)$ and $e$ is optimal against $\left(\mu_{1}, \mu_{3}\right)$. This, in turn, implies that $\mu_{3}(g) \geq 2 / 3$ and $\mu_{3}(h) \geq 2 / 3$, which is clearly impossible. Hence, $g$ is not a Nash strategy. 


\begin{tabular}{|l|l|l|l|}
\hline$g$ & $d$ & $e$ & $f$ \\
\hline$a$ & $0,0,3$ & $0,0,3$ & $3,3,3$ \\
\hline$b$ & $0,0,0$ & $0,0,0$ & $3,3,3$ \\
\hline$c$ & $3,3,3$ & $3,3,3$ & $3,3,3$ \\
\hline
\end{tabular}

\begin{tabular}{|l|l|l|l|}
\hline$h$ & $d$ & $e$ & $f$ \\
\hline$a$ & $0,0,2$ & $0,0,0$ & $0,0,0$ \\
\hline$b$ & $0,0,0$ & $0,0,2$ & $0,0,0$ \\
\hline$c$ & $0,0,0$ & $0,0,0$ & $0,0,0$ \\
\hline
\end{tabular}

\begin{tabular}{|l|l|l|l|}
\hline$i$ & $d$ & $e$ & $f$ \\
\hline$a$ & $0,0,0$ & $0,0,0$ & $3,3,3$ \\
\hline$b$ & $0,0,3$ & $0,0,3$ & $3,3,3$ \\
\hline$c$ & $3,3,3$ & $3,3,3$ & $3,3,3$ \\
\hline
\end{tabular}

Fig. 7 CIB and belief in CIB cannot be dropped

Dropping CIB: Consider the three-player game in Fig. 7. Construct an epistemic model $\mathcal{M}$ such that $T_{1}=\left\{t_{1}\right\}, T_{2}=\left\{t_{2}\right\}, T_{3}=\left\{t_{3}\right\}, \beta_{1}\left(t_{1}\right)$ assigns probability $1 / 2$ to $\left(\left(d, t_{2}\right),\left(h, t_{3}\right)\right)$ and probability $1 / 2$ to $\left(\left(e, t_{2}\right),\left(h, t_{3}\right)\right), \beta_{2}\left(t_{2}\right)$ assigns probability $1 / 2$ to $\left(\left(a, t_{1}\right),\left(h, t_{3}\right)\right)$ and probability $1 / 2$ to $\left(\left(b, t_{1}\right),\left(h, t_{3}\right)\right)$, and $\beta_{3}\left(t_{3}\right)$ assigns probability $1 / 2$ to $\left(\left(a, t_{1}\right),\left(d, t_{2}\right)\right)$ and probability $1 / 2$ to $\left(\left(b, t_{1}\right),\left(e, t_{2}\right)\right)$. Then, $t_{3}$ does not have CIB. It may be verified that $t_{3}$ satisfies the other six conditions.

Strategy $h$ is rational for $t_{3}$. However, we will show that $h$ is not a Nash strategy. Assume, namely, that $\left(\mu_{1}, \mu_{2}, \mu_{3}\right)$ would be a Nash equilibrium such that $h$ would be optimal against $\left(\mu_{1}, \mu_{2}\right)$. If $\mu_{1}(a) \leq 1 / 2$, it can be shown that $u_{3}\left(h, \mu_{1}, \mu_{2}\right)<$ $u_{3}\left(i, \mu_{1}, \mu_{2}\right)$. If $\mu_{1}(a) \geq 1 / 2$, it can be shown that $u_{3}\left(h, \mu_{1}, \mu_{2}\right)<u_{3}\left(g, \mu_{1}, \mu_{2}\right)$. Hence, $h$ can never be optimal against $\left(\mu_{1}, \mu_{2}\right)$, and therefore $h$ is not a Nash strategy.

Dropping belief in CIB: Consider again the three-player game in Fig. 7. Construct an epistemic model $\mathcal{M}$ such that $T_{1}=\left\{t_{1}\right\}, T_{2}=\left\{t_{2}\right\}, T_{3}=\left\{t_{3}\right\}, \beta_{1}\left(t_{1}\right)$ assigns probability $1 / 2$ to $\left(\left(d, t_{2}\right),\left(h, t_{3}\right)\right)$ and probability $1 / 2$ to $\left(\left(e, t_{2}\right),\left(h, t_{3}\right)\right), \beta_{2}\left(t_{2}\right)$ assigns probability $1 / 2$ to $\left(\left(a, t_{1}\right),\left(h, t_{3}\right)\right)$ and probability $1 / 2$ to $\left(\left(b, t_{1}\right),\left(h, t_{3}\right)\right)$, and $\beta_{3}\left(t_{3}\right)$ assigns probability $1 / 2$ to $\left(\left(a, t_{1}\right),\left(d, t_{2}\right)\right)$ and probability $1 / 2$ to $\left(\left(b, t_{1}\right),\left(e, t_{2}\right)\right)$. Then, $t_{1}$ does not believe in CIB, since $t_{3}$ does not have CIB. It may be verified that $t_{1}$ satisfies the other six conditions.

Strategy $a$ is rational for $t_{1}$. However, we will show that $a$ is not a Nash strategy. We have seen above that $h$ is not a Nash strategy. In particular, there is no Nash equilibrium that assigns positive probability to $h$. But then, $a$ cannot be optimal in a Nash equilibrium, and hence $a$ is not a Nash strategy.

\section{Discussion of main result}

Our main result comes in two parts: In Theorem 4.5 we first show that, if a player satisfies BOR, SRB and CIB, and believes that every opponent satisfies BOR, SRB, $\mathrm{PB}$ and CIB, then this player will eventually choose a Nash strategy. We next prove in Theorem 4.7 that every Nash strategy for player $i$ can be supported by a player $i$ belief hierarchy that satisfies these seven conditions. By combining these two results, we may thus view the seven conditions above as a one-person doxastic characterization of Nash strategies.

\subsection{Discussion of SRB and PB}

Since SRB and PB are new, and play a crucial role in the doxastic characterization of Nash strategies, we will discuss these two conditions in some more depth here. Both SRB and PB reflect the event that player $i$ uses his own beliefs as a "focal point" for what he believes that others believe. In SRB, namely, player $i$ uses his own belief 
about himself (assuming that he has a correct belief about himself) as a focal point for what he believes that others believe about himself, whereas in PB player $i$ uses his own belief about opponent $j$ as a focal point for what he believes that others believe about opponent $j$. Now, can it be reasonable, at an intuitive level, to take your own belief as a focal point for what you believe that others believe? In my opinion, this can be reasonable if there are no serious hints that some of your opponents really reason differently than you do. This may happen if you have little or no information about the opponents' characteristics, and in particular about their ways of reasoning. In such situations, one "easy" way to form a belief about what others believe is simply to assume that your opponents have the same beliefs as you do. Moreover, if your own belief satisfies certain properties, such as BOR, then by believing that others have the same belief as you do, you will be sure to believe that others will share these properties too. Hence, if you face a serious lack of information about your opponents' beliefs, then choosing your own belief as a "focal candidate" for the beliefs of others may make intuitive sense. This lack of information about others will often occur in the type of setting we consider, namely static games in which the game is only played once, and players do not communicate with each other before reaching a decision.

\subsection{Can SRB be weakened?}

In Sect. 5 we have seen that none of the seven conditions in Theorem 4.5 can be dropped completely. In particular, by completely dropping the condition that player $i$ has SRB, or believes that every opponent has SRB, one can no longer guarantee that player $i$ will choose a Nash strategy. However, can we replace the notion of SRB in Theorem 4.5 by a weaker requirement without violating the content of the theorem? The answer is "yes." Consider, namely, the weaker condition stating that $t_{i}$ believes that every opponent holds a correct belief about player $i$ 's belief about his opponents' choices. Let us call this condition weakly self-referential beliefs (WSRB). The difference with SRB is that the latter requires that $t_{i}$ believes that every opponent holds a correct belief about player $i$ 's complete belief hierarchy, not only about his belief about the opponents' choices. One can show the following result by basically copying the proof from Theorem 4.5.

Theorem 6.1 Let $\mathcal{M}=\left(T_{i}, \beta_{i}\right)_{i \in I}$ be a finite epistemic model for a finite game $\Gamma$. Let $t_{i} \in T_{i}$ be a type that satisfies BOR, WSRB and CIB, and believes that every opponent satisfies BOR, WSRB, PB and CIB. Then, every strategy that is rational for $t_{i}$ is a Nash strategy for $\Gamma$.

\subsection{Single player's perspective}

Remember that our Theorem 4.5 only imposes conditions on the beliefs of a single player, namely player $i$. These conditions guarantee that player $i$ will choose a Nash strategy. Moreover, as we have seen in Corollary 4.6, the same conditions imply that player $i$ 's belief about his opponents' choices, together with his belief about the opponents' belief about his own choice, constitute a Nash equilibrium. In this context, a 


\begin{tabular}{|l|l|l|}
\hline & $c$ & $d$ \\
\hline$a$ & 1,1 & 0,0 \\
\hline$b$ & 0,0 & 1,1 \\
\hline
\end{tabular}

Fig. 8 Beliefs of different players may not constitute a Nash equilibrium

Nash equilibrium is interpreted as a "personal object" that is completely inside player $i$ 's mind.

Now, suppose there are only two players in a game, player $i$ and player $j$, who both satisfy the conditions in Theorem 4.5. Do the players' beliefs about the opponent's choice then constitute a Nash equilibrium? The answer is "no." Although the conditions guarantee that player $i$ 's personal beliefs about player $j$ constitute a Nash equilibrium (in the sense of Corollary 4.6) and that player $j$ 's personal beliefs about player $i$ constitute a Nash equilibrium, it may well be that both players have different Nash equilibria in their minds. Consequently, $i$ 's belief about $j$ 's choice and $j$ 's belief about $i$ 's choice may not constitute a Nash equilibrium in beliefs. Consider, for instance, the coordination game in Fig. 8. Construct an epistemic model $\mathcal{M}$ such that $T_{1}=\left\{t_{1}^{a}, t_{1}^{b}\right\}, T_{2}=\left\{t_{2}^{c}, t_{2}^{d}\right\}, \beta_{1}\left(t_{1}^{a}\right)$ assigns probability 1 to $\left(c, t_{2}^{c}\right), \beta_{1}\left(t_{1}^{b}\right)$ assigns probability 1 to $\left(d, t_{2}^{d}\right), \beta_{2}\left(t_{2}^{c}\right)$ assigns probability 1 to $\left(a, t_{1}^{a}\right)$ and $\beta_{2}\left(t_{2}^{d}\right)$ assigns probability 1 to $\left(b, t_{1}^{b}\right)$. Then, every type in $T_{1}$ and $T_{2}$ satisfies the conditions in Theorem 4.5 , however $t_{1}^{a}$ 's belief about 2's choice and $t_{2}^{d}$ 's belief about 1 's choice do not constitute a Nash equilibrium.

In this respect, our Theorem 4.5 differs crucially from Aumann and Brandenburger (1995), Asheim (2006) and Brandenburger and Dekel (1987) who impose conditions on the beliefs of all players which, together, imply that the players' beliefs about the opponents' choices constitute a Nash equilibrium. In the following section, we will explore the differences with these models in some more detail.

\section{Comparison with other models}

\subsection{Aumann and Brandenburger's model}

Aumann and Brandenburger (1995) (AB from now on) make a distinction between the case of two players and the case of more than two players, and provide sufficient conditions for Nash equilibrium for both cases. In AB's model, a type for player $i$ does not only specify $i$ 's belief hierarchy, but also $i$ 's strategy choice and $i$ 's utility function. It is therefore possible to say that type $t_{i}$ is rational. AB's theorem for two-player games may be formulated as follows: Consider a pair $\left(u_{1}, u_{2}\right)$ of utility functions, a pair $\left(\mu_{1}, \mu_{2}\right) \in \Delta\left(S_{1}\right) \times \Delta\left(S_{2}\right)$ of probability distributions over strategy choices, and a pair $\left(t_{1}, t_{2}\right)$ of types. If at $\left(t_{1}, t_{2}\right)$ it is true that (1) both players are rational and believe that the opponent is rational, (2) both players $i$ have utility function $u_{i}$ and believe that opponent $j$ has utility function $u_{j}$, and (3) both players $i$ have belief $\mu_{j}$ 
about $j$ 's strategy choice, and believe that $j$ has belief $\mu_{i}$ about $i$ 's strategy choice, then $\left(\mu_{1}, \mu_{2}\right)$ is a Nash equilibrium with respect to $\left(u_{1}, u_{2}\right)$.

An important difference with our model is that $\mathrm{AB}$ simultaneously impose conditions on the belief hierarchies of both players, whereas our model only imposes conditions on the beliefs of a single player. So, in this sense AB's model is more restrictive. However, if we concentrate on the conditions that $\mathrm{AB}$ impose on the beliefs of a single player, we see that these are weaker than our conditions in Theorem 4.5 for two-player games. For instance, condition (1) above implies that player $i$ BOR, but not necessarily that $i$ believes that $j$ BOR, as we impose in Theorem 4.5. Also, condition (3) above does not imply that $i$ has SRB: $i$ may have belief $\mu_{j}$ about $j$ 's choice, and at the same time believe that $j$ believes that $i$ has a different belief about $j$ 's choice. So, in fact, condition (3) does not even imply that $i$ has WSRB (see Theorem 6.1). Hence, when analyzed from a single player's perspective only, AB's conditions are weaker than our conditions for two-player games.

In order to highlight the differences with our model, consider again the game from Fig. 8. In terms of AB's model, let $T_{1}=\left\{t_{1}^{a}, \hat{t}_{1}^{a}\right\}$ and $T_{2}=\left\{t_{2}^{c}, \hat{t}_{2}^{c}, t_{2}^{d}\right\}$ be sets of types where $t_{1}^{a}, \hat{t}_{1}^{a}$ choose $a, t_{2}^{c}$ and $\hat{t}_{2}^{c}$ choose $c, t_{2}^{d}$ chooses $d, t_{1}^{a}$ believes that 2 's type is $\hat{t}_{2}^{c}$, $\hat{t}_{1}^{a}$ believes that 2's type is $t_{2}^{d}, t_{2}^{c}$ believes that 1 's type is $t_{1}^{a}, \hat{t}_{2}^{c}$ believes that 1 's type is $\hat{t}_{1}^{a}$ and $t_{2}^{d}$ believes that 1's type is $t_{1}^{a}$. See Fig. 9 for an illustration. Here, the arrows denote beliefs. For instance, the arrow from $t_{1}^{a}$ to $\hat{t}_{2}^{c}$ means that type $t_{1}^{a}$ believes that player 2's type is $\hat{t}_{2}^{c}$. Furthermore, assume that each type has the utility function as depicted in Fig. 8. Let $\mu_{1}$ be the probability distribution that assigns probability 1 to $a$, and let $\mu_{2}$ be the probability distribution that assigns probability 1 to $c$. Then at $\left(t_{1}^{a}, t_{2}^{c}\right)$ conditions (1), (2) and (3) above are satisfied. That is, at $\left(t_{1}^{a}, t_{2}^{c}\right)$ AB's sufficient conditions for Nash equilibrium are met. However, type $t_{1}^{a}$ does not believe that player 2 BOR. Namely, $t_{1}^{a}$ believes that player 2 believes that player 1 is of type $\hat{t}_{1}^{a}$ and chooses $a$. Type $\hat{t}_{1}^{a}$, in turn, believes that player 2 chooses $d$, and hence $a$ is not rational for type $\hat{t}_{1}^{a}$. So, $t_{1}^{a}$ believes that player 2 believes that player 1 chooses irrationally. Also, $t_{1}^{a}$ does not have SRB: $t_{1}^{a}$ believes that player 2 chooses $c$, whereas $t_{1}^{a}$ believes that player 2 believes that player 1 is of type $\hat{t}_{1}^{a}$ who believes that player 2 chooses $d$. In fact, $t_{1}^{a}$ does not even have WSRB. Hence, type $t_{1}^{a}$ does not satisfy our sufficient conditions for Nash strategy choice, although AB's conditions are met at $\left(t_{1}^{a}, t_{2}^{c}\right)$.

The example above shows, in particular, that AB's conditions do not imply common belief in rationality at $\left(t_{1}, t_{2}\right)$. Here, by common belief in rationality, at $\left(t_{1}, t_{2}\right)$ we mean that both players are rational at $\left(t_{1}, t_{2}\right)$, both players believe at $\left(t_{1}, t_{2}\right)$ that both players are rational, and so on. Polak (1995) proves that, if conditions (2) and (3)

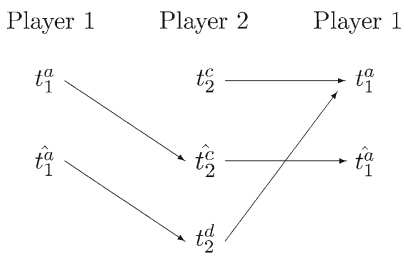

Fig. 9 An example illustrating AB's theorem 
above are strengthened by imposing common belief in the conjectures $\left(\mu_{1}, \mu_{2}\right)$ and the utility functions $\left(u_{1}, u_{2}\right)$, then common belief in rationality will hold at $\left(t_{1}, t_{2}\right)$. Brandenburger and Dekel (1989) have shown a related result for two-player games: If there is common belief in the conjectures $\left(\mu_{1}, \mu_{2}\right)$ and the utility functions $\left(u_{1}, u_{2}\right)$, then $\left(\mu_{1}, \mu_{2}\right)$ is a Nash equilibrium if and only if common belief in rationality holds.

For the case of more than two players, AB add the following two conditions: (4) at the type profile $\left(t_{i}\right)_{i \in I}$ the types' belief hierarchies are derived from a common prior probability distribution on the set of type profiles, and (5) at $\left(t_{i}\right)_{i \in I}$ there is common belief in the profile $\left(\beta_{i}\right)_{i \in I} \in \times_{i \in I} \Delta\left(S_{-i}\right)$ of beliefs about the opponents' strategies. By the latter we mean that $t_{i}$ 's belief about the opponents' choices is $\beta_{i}$, that $t_{i}$ believes that every opponent $j$ has belief $\beta_{j}$ about the other players' choices, that $t_{i}$ believes that every opponent $j$ believes that every other player $k$ has belief $\beta_{k}$ about the opponents' choices, and so on. By adding these conditions (4) and (5), AB are able to show that there is some profile $\left(\mu_{i}\right)_{i \in I} \in \times_{i \in I} \Delta\left(S_{i}\right)$ of probability distributions over strategy choices such that for every player $i$, type $t_{i}$ 's belief about the opponents' choices is given by $\left(\mu_{j}\right)_{j \neq i}$, and that $\left(\mu_{i}\right)_{i \in I}$ is a Nash equilibrium with respect to $\left(u_{i}\right)_{i \in I}$. In particular, the conditions (4) and (5) imply that $i$ 's belief about $j$ 's choice is stochastically independent from $i$ 's belief about $k$ 's choice, and that two different players $i$ and $j$ have the same belief about $k$ 's choice. These two properties are crucial for their proof. In our model, these two properties follow from the assumption that $t_{i}$ has SRB and CIB, and believes that every opponent has SRB, PB and CIB (see Lemma 4.4). Hence, one could say that in our model the conditions of CIB and belief in $\mathrm{PB}$ and CIB play a similar role as the conditions (4) and (5) in AB.

\subsection{Asheim's model}

Asheim (2006, p. 5), provides a sufficient condition for Nash equilibrium for the case of two players. He basically uses the same epistemic model as we do, and his result may be stated as follows: Consider a pair $\left(t_{1}, t_{2}\right)$ of types, and assume that (1) $t_{1}$ and $t_{2}$ BOR, and (2) $t_{1}$ believes that 2's type is $t_{2}$, and $t_{2}$ believes that 1 's type is $t_{1}$. Then, $t_{1}$ 's belief about 2's choice and $t_{2}$ 's belief about 1's choice constitute a Nash equilibrium.

Similar to AB, Asheim simultaneously imposes conditions on the belief hierarchies of both players. However, if we analyze Asheim's conditions from a single-player perspective, then we see that Asheim's conditions imply ours, Focus, namely, on a single player, say player $i$. Conditions (1) and (2) above together imply that $t_{i} \mathrm{BOR}$, and that $t_{i}$ believes that $j \mathrm{BOR}$, since $t_{i}$ believes that $j$ 's type is $t_{j}$, who is supposed to BOR. Moreover, conditions (1) and (2) imply that $t_{i}$ has SRB and believes that $j$ has SRB. Namely, $t_{i}$ believes that $j$ 's type is $t_{j}$, who believes that $i$ 's type is $t_{i}$. Hence, $t_{i}$ believes that $j$ believes that $i$ 's type is $t_{i}$, and hence $t_{i}$ has SRB. Also, by the same reasoning, $t_{j}$ has SRB. Since $t_{i}$ believes that $j$ 's type is $t_{j}$, type $t_{i}$ believes that $j$ has SRB. Summarizing, we may conclude that Asheim's conditions (1) and (2) imply that $t_{i}$ BOR, believes that $j$ BOR, has SRB, and believes that $j$ has SRB. So, Asheim's conditions, when analyzed from a single-player's perspective, imply our sufficient conditions for Nash strategy choice.

Tan and Werlang (1988), in their Theorem 6.2.1, p. 382, provide conditions for Nash equilibrium in two-player games that are similar to Asheim's, although somewhat 
stronger. They assume, like Asheim, that $t_{1}$ believes that 2's type is $t_{2}$, and that $t_{2}$ believes that 1 's type is $t_{1}$. However, Tan and Werlang impose common belief in rationality at $\left(t_{1}, t_{2}\right)$, instead of only requiring that $t_{1}$ and $t_{2} \mathrm{BOR}$.

\subsection{Brandenburger and Dekel's model}

Brandenburger and Dekel (1987) (BD from now on) use a model which substantially differs from ours and the ones above. BD assume that there is a finite state space $\Omega$, that every player $i$ holds a prior belief $P_{i} \in \Delta(\Omega)$, that for every player $i$ there is an information partition $\mathcal{H}_{i}$ of $\Omega$, and that for every $H_{i} \in \mathcal{H}_{i}$ there is a conditional belief $P_{i}\left(\cdot \mid H_{i}\right) \in \Delta\left(H_{i}\right)$ which is derived from $P_{i}$ by Bayes' rule whenever possible. A strategy map for player $i$ is an $\mathcal{H}_{i}$-measurable map $f_{i}$ from $\Omega$ to $S_{i}$. A profile $\left(f_{i}\right)_{i \in I}$ of strategy maps is called an a posteriori equilibrium if for every player $i$, at every $H_{i} \in \mathcal{H}_{i}$ the prescribed strategy is optimal given the conditional belief $P_{i}\left(\cdot \mid H_{i}\right)$ and the opponents' strategy maps $\left(f_{j}\right)_{j \neq i}$. Hence, it is implicitly assumed that players have correct beliefs about the opponents' strategy maps, and that there is common belief in this event. The prior beliefs $\left(P_{i}\right)_{i \in I}$ are called concordant if, for every choice of the strategy maps, two different players $i$ and $j$ have the same prior belief about $k$ 's choice. The information partitions $\left(\mathcal{H}_{j}\right)_{j \neq i}$ are called $P_{i}$-conditionally independent if, for every choice of the strategy maps, $i$ 's belief about $j$ 'choice is independent from $i$ 's belief about $k$ 's choice. The information partitions $\left(\mathcal{H}_{j}\right)_{j \neq i}$ are called $P_{i}$-informationally independent if, for every choice of the strategy maps, $i$ 's belief about the opponents' choices does not depend on the information set $H_{i}$.

BD's theorem on page 1401 can now be stated as follows: suppose that the prior beliefs $\left(P_{i}\right)_{i \in I}$ and the information partitions $\left(\mathcal{H}_{i}\right)_{i \in I}$ are such that (1) the prior beliefs $\left(P_{i}\right)_{i \in I}$ are concordant, and for every $i$, the information partitions $\left(\mathcal{H}_{j}\right)_{j \neq i}$ are (2) $P_{i^{-}}$ conditionally independent and (3) $P_{i}$-informationally independent. Then, for every a posteriori equilibrium there is a profile $\left(\mu_{i}\right)_{i \in I} \in \times_{i \in I} \Delta\left(S_{i}\right)$ of probability distributions over strategy choices such that, for every $i$, player $i$ 's prior belief about the opponents' choices is given by $\left(\mu_{j}\right)_{j \neq i}$, and $\left(\mu_{i}\right)_{i \in I}$ is a Nash equilibrium.

In BD's theorem, selecting an a posteriori equilibrium implies that player $i$ BOR. Concordance of the prior beliefs guarantees that $i$ and $j$ have the same beliefs about $k$ 's choice, and therefore implies our condition of PB. The assumption in BD that the information partitions $\left(\mathcal{H}_{j}\right)_{j \neq i}$ are $P_{i}$-informationally independent guarantees that $i$ 's belief about his opponents is independent of his information state, and therefore $i$ believes that his opponents are right about his own beliefs. (Recall the implicit assumption that players have correct beliefs about the opponents' strategy maps, and that there is common belief in this event). The assumption of $P_{i}$-informational independence therefore implies that player $i$ has SRB. In BD, $P_{i}$-conditional independence guarantees that $i$ 's belief about $j$ 's choice is independent from $i$ 's belief about $k$ 's choice. Since $P_{j}$ - and $P_{k}$-informational independence implies that $i$ only deems one belief hierarchy, and hence only one type, possible for opponents $j$ and $k, P_{i}$-conditional independence and $P_{j}$ - and $P_{k}$-informational independence together imply that player $i$ has CIB. Summarizing, we see that BD's conditions imply that player $i$ BOR, and has SRB, PB and CIB. Now, fix an opponent $j$. By BD's conditions, player $j$ also BOR, 
and has SRB, PB and CIB. Since BD's condition of $P_{j}$-informational independence implies that player $i$ is correct about $j$ 's belief hierarchy, it follows that $i$ believes that every opponent $j$ BOR, and has SRB, PB and CIB. We may thus conclude that BD's conditions, when analyzed from a single-player's perspective, imply ours.

Acknowledgements I wish to thank Geir Asheim and two referees for their helpful suggestions.

\section{References}

Asheim, G. B. (2006). The consistent preferences approach to deductive reasoning in games. Theory and Decision Library. Dordrecht: Springer.

Aumann, R., \& Brandenburger, A. (1995). Epistemic conditions for Nash equilibrium. Econometrica, 63, 1161-1180.

Bernheim, D. (1984). Rationalizable strategic behavior. Econometrica, 52, 1007-1028.

Brandenburger, A., \& Dekel, E. (1987). Rationalizability and correlated equilibria. Econometrica, 55, 1391-1402.

Brandenburger, A., \& Dekel, E. (1989). The role of common knowledge assumptions in game theory. In F. Hahn (Ed.), The economics of missing markets, information and games (pp. 46-61). Oxford: Oxford University Press.

Brandenburger, A., \& Friedenberg, A. (2006). Intrinsic correlation in games. Available at http:// pages.stern.nyu.edu/ abranden/

Myerson, R. (1991). Game theory: Analysis of conflict. Cambridge, Massachusetts: Harvard University Press.

Nash, J. (1951). Non-cooperative games. Annals of Mathematics, 54, 286-295.

Pearce, D. (1984). Rationalizable strategic behavior and the problem of perfection. Econometrica, 52, 1029-1050.

Polak, B. (1995). Epistemic conditions for Nash equilibrium, and common knowledge of rationality. Econometrica, 67, 673-676.

Tan, T., \& Werlang, S. R. C. (1988). The bayesian foundations of solution concepts of games. Journal of Economic Theory, 45, 370-391. 